\title{
Hotel Reservation System Based Local Area Network at Samarinda
}

\author{
Heny Pratiwi \\ Teknik Informatika, STMIK Widya Cipta Dharma, Samarinda, Indonesia \\ henypratiwi@wicida.ac.id
}

\begin{abstract}
The Hotel industry is one of the most vibrant businesses today, however, the current hotel reservation system is still largely unfamiliar with computerized processes. The process of logging in and outgoing guests still use simple office applications without formulas and without database-based. Any receipt of payment submitted to the guest also cannot be automatically printed from the office application. Every month an incoming and outgoing guest report will be made abased on notes from the hotel reservation. The hotel reservation system will be built using Visual Basic 6.0 and network-based programming languages. In this study, data collection techniques used are literature study and documentation, interviews, observation, data analysis, needs analysis, and technology analysis. Analysis methods and design system used in this research are Flow of Document (FOD) and Data Flow Diagram (DFD). Testing the system using black box testing method, by testing the program interface to analyze the input and do all the functional requirements of the program.
\end{abstract}

Keywords-system; reservation; hotel; local area network

\section{INTRODUCTION}

Hotel reservation system based Local Area Network is a computerized system with handling hotel problems that exist in Samarinda. The hotel-based hotel reservation system includes data collection of incoming and outgoing guest activities. Now, the hotel's on-going reservation system, has not used a computerized process. The process that occurs is logging in and outgoing guests still use simple office applications without formulas and without database-based.

Any bills of payment submitted to the guest also cannot be automatically printed from the office application. Every month an incoming guest report will be made from the hotel reservation process that has been done as well as reports on the activities of incoming guests and guests out at the hotel. Based on the current system, there are still problems such as the need for a long time in terms of the accumulated use of laundry services and restaurants used by guests. Because of that, it need help to solve the problems encountered, made a hotel-based reservation system network. With this system, we expected to handle the constraints faced in the hotel reservation system a, make it easier for the administration in guest data processing, transactions incoming and outgoing guests and printing reports required by the management.

\section{LITERATURE REVIEW}

\section{A. Hotel Reservation}

A hotel reservation system, commonly known as a central reservation system (CRS) is a computerized system that stores and distributes information of a hotel, resort or other lodging facilities [1].

\section{B. Local Area Network}

LANs connect computers and peripheral devices in a limited physical area, such as a business, office, a limited physical area, such as a business, office, laboratory or college campus by means of permanent links (wires) that transmit data rapidly [2].

A local area network (LAN) is a communication network that interconnects a variety of data communicating devices within a small geographic area and broadcasts data at high data transfer rates with very low error rates. Since the local area network first appeared in the 1970s, its use has become widespread in commercial and academic environments.

A LAN is a high-speed data network that covers a relatively small geographic area. It typically connects workstations, personal computers, printers, servers, and other devices. LANs offer computer users many advantages, including shared access to devices and applications, file exchange between connected users, and communication between users via electronic mail and other applications [3].

A LAN consists of two or more personal computer, printers and high capacity disk storage device called printers and high capacity disk storage device called file servers, which enable each computer on the file servers, which enable each computer on the network to access a common of files.

LAN topologies define the manner in which network devices are organized. Four common LAN topologies exist: bus, ring, star, and tree. These topologies are logical architectures, but the actual devices need not be physically organized in these configurations. Logical bus and ring topologies, for example, are commonly organized physically as a star. A bus topology is a linear LAN architecture in which transmissions from network stations propagate the length of the medium and are received by all other stations. Of the three most widely used LAN implementations, Ethernet/IEEE 802.3 networks - including 100BaseT-implement a bus topology, which is illustrated in Figure 1. 


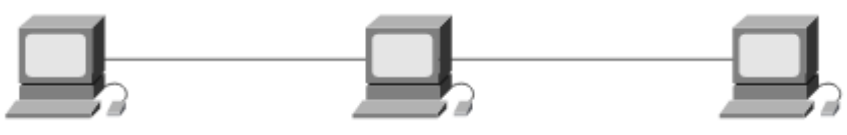

Fig. 1. Some Networks Implement a Local Bus Topology

A ring topology is a LAN architecture that consists of a series of devices connected to one another by unidirectional transmission links to form a single closed loop. Both Token Ring/IEEE 802.5 and FDDI networks implement a ring topology. Figure 2 depicts a logical ring topology.

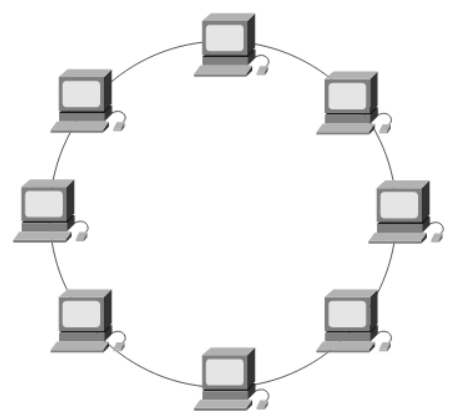

Fig. 2. Some Networks Implement a Local Ring Topology

A star topology is a LAN architecture in which the endpoints on a network are connected to a common central hub, or switch, by dedicated links. Logical bus and ring topologies are often implemented physically in a star topology, which is illustrated in Figure 3.

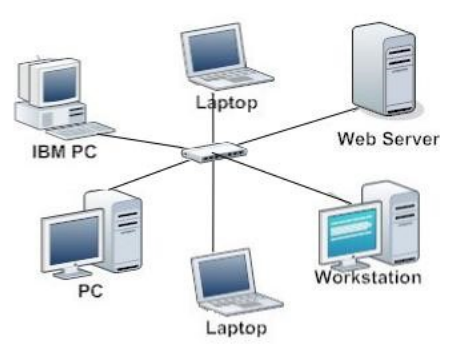

Fig. 3. Some Networks Implement a Local Star Topology

A tree topology is a LAN architecture that is identical to the bus topology, except that branches with multiple nodes are possible in this case. Figure 4 illustrates a logical tree topology.

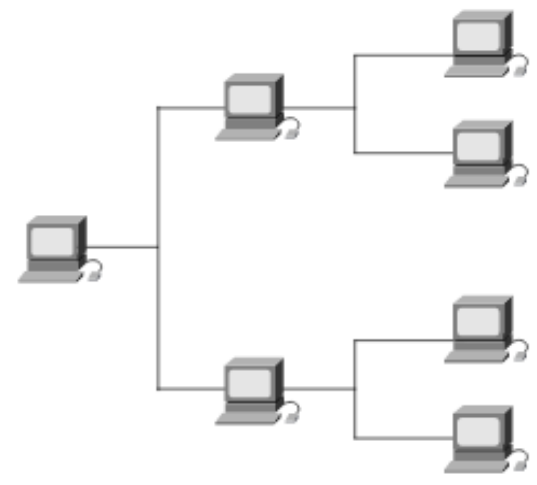

Fig. 4. A Logical Tree Topology Can Contain Multiple Nodes

\section{Prototyping Model}

Prototype Model places more effort in creating the actual software instead of concentrating on documentation. This way, the actual software could be released in advance. Prototyping requires more user involvement and allows them to see and interact with a prototype allowing them to provide better and more complete feedback and specifications. The presence of the prototype being examined by the user prevents many misunderstandings that occur when each side believe the other understands what they said. The final product is more likely to satisfy the user's desire for look, feel and performance [4].

A prototype is part of a product that express the logic and physical external interface to display. Potential consumers use prototypes and provide input to the development team before large scale development begins. Seeing and believing are the things that are expected to be achieved in the prototype. Using this approach, consumers and development teams can clarify their needs and interpretations. The stages in the prototype are as follows.

\section{Analysis / collection needs}

Customers and developers together define the entire software format, identify all needs, and outline the system to be created.

\section{Building prototyping.}

Build prototyping by creating temporary designs that focus on serving to customers (e.g. by creating input and output formats).

\section{Evaluation of prototyping}

This evaluation is done by the customer whether the prototyping that has been built is in accordance with the wishes of customers. If it is appropriate then step 4 will be taken. Otherwise prototyping is revised by repeating steps 1,2 , and 3.

4. Encoding the system

In this stage the agreed prototyping is translated into the appropriate programming language.

5. Test the system

Once the system has become a ready-made software, it should be tested before use. This test is done by White Box, Black Box, Path Base, or architecture testing and others.

\section{System Evaluation}

The customer evaluates whether the ready-made system is as expected. If yes step 7 is done, otherwise repeat steps 4 and 5 .

7. Application of the system

The software that the customer has tested and received is ready to use.

\section{Flow Of Diagram}

Flow of Document (FOD) is a tool that describes the flow of data through the system and its work or processes performed by the system [5]. 
TABLE I. FLOW OF DOCUMENT SYMBOL

\begin{tabular}{|l|c|}
\hline & NAME \\
\hline & Document \\
\hline & Multi Document \\
\hline & Manual Process \\
\hline & Computer Process \\
\hline & Orchieve \\
\hline
\end{tabular}

\section{E. Database}

Database is any collection of related data. A database also a persistent, logically coherent collection of inherently meaningful data, relevant to some aspects of the real world. A database management system (DBMS) is a collection of programs that enables users to create and maintain a database [6].

Database Management Systems provide several functions in addition to simple file management:

- allow concurrency
- control security

- maintain data integrity

- provide for backup and recovery

- control redundancy

- allow data independence

- provide non-procedural query language

- perform automatic query optimization

Many different individuals are involved with a database management system over its life: systems analysts, database designers, database administrators, application developers, and users.

III. Design And Development of the Proposed System

A. The Existing System

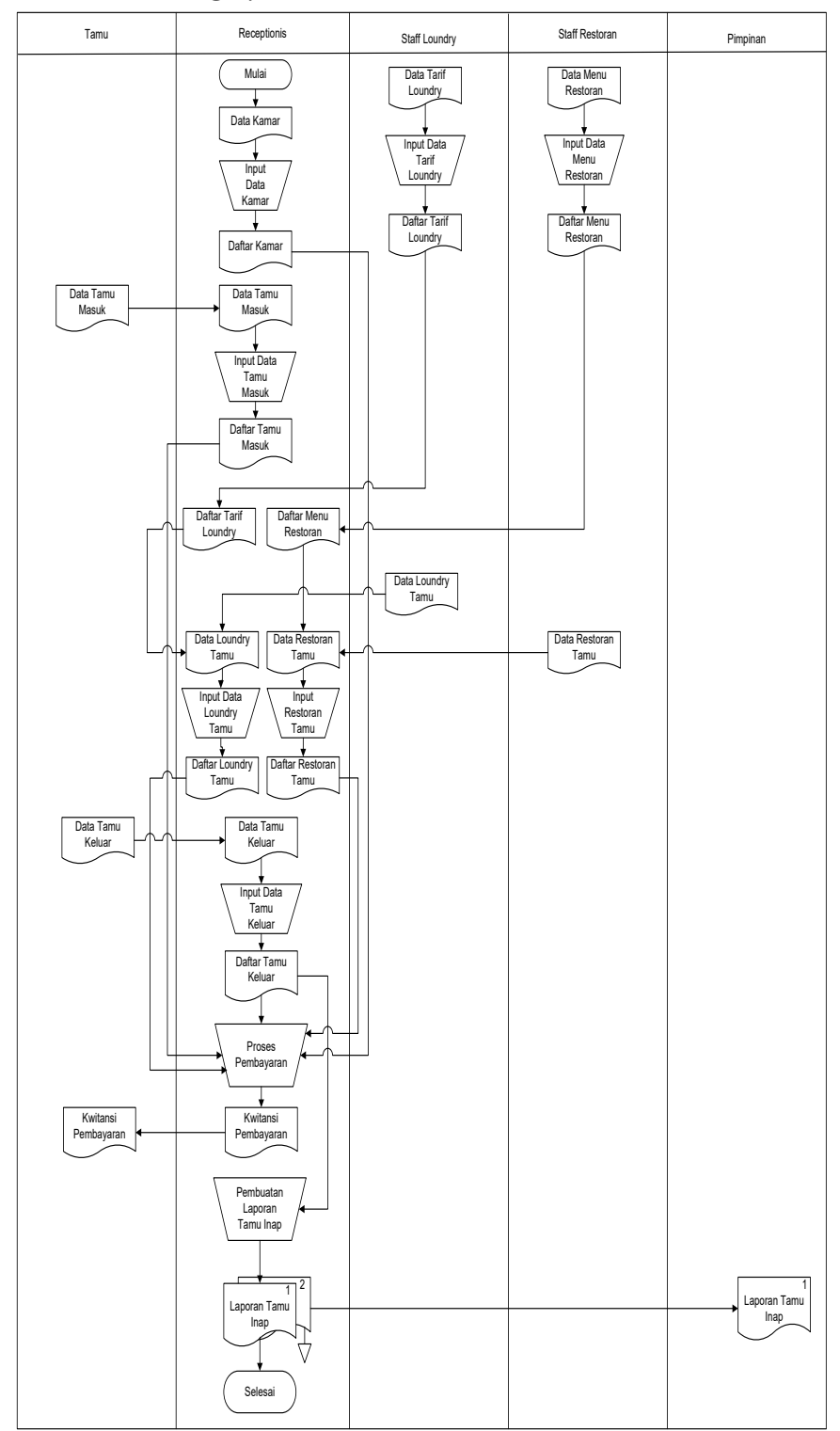

Fig. 5. Existing system of hotel reservation 


\section{B. The Proposed System}

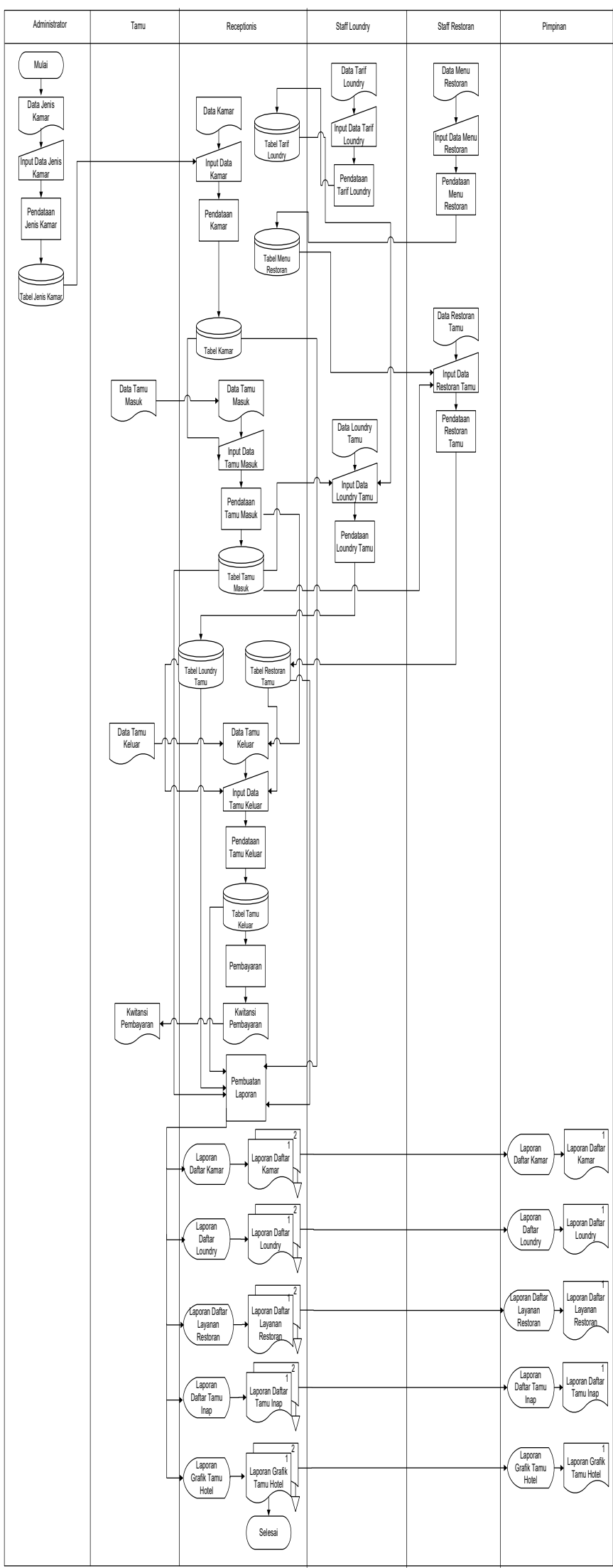

Fig. 6. Proposed hotel reservation system

\section{Context Diagram}

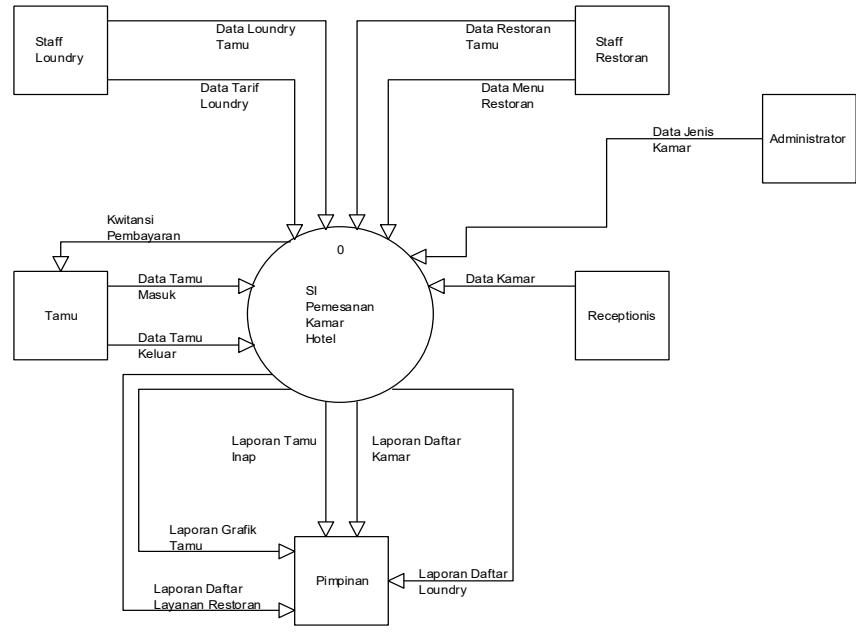

Fig. 7. Context Diagram of hotel reservation system

D. Data Flow Diagram (DFD) Level 0

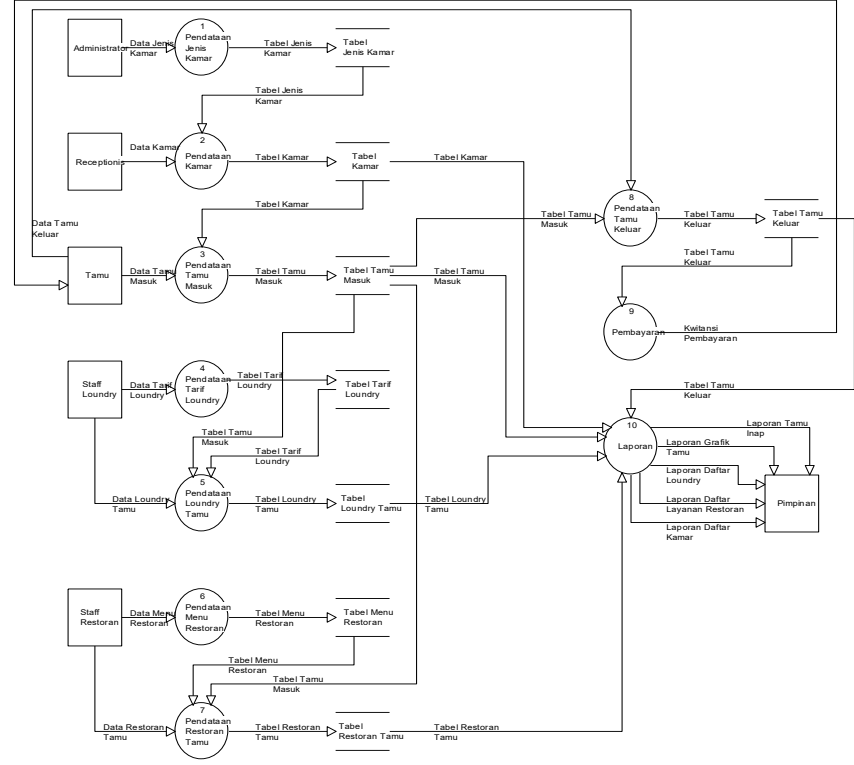

Fig. 8. DFD Level 0 of hotel reservation system

E. Data Flow Diagram (DFD) Level 1

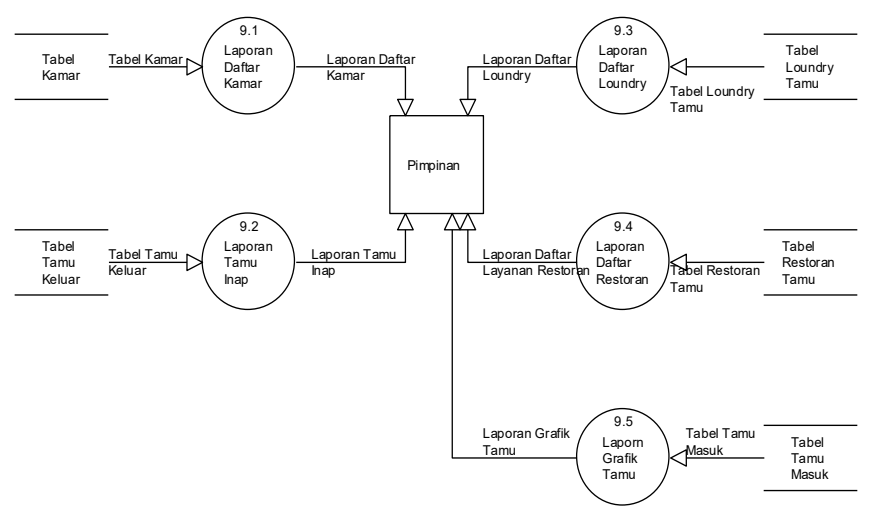

Fig. 9. DFD Level 1 of hotel reservation system 


\section{F. Hierarchy Plus Input-Process-Output (HIPO)}

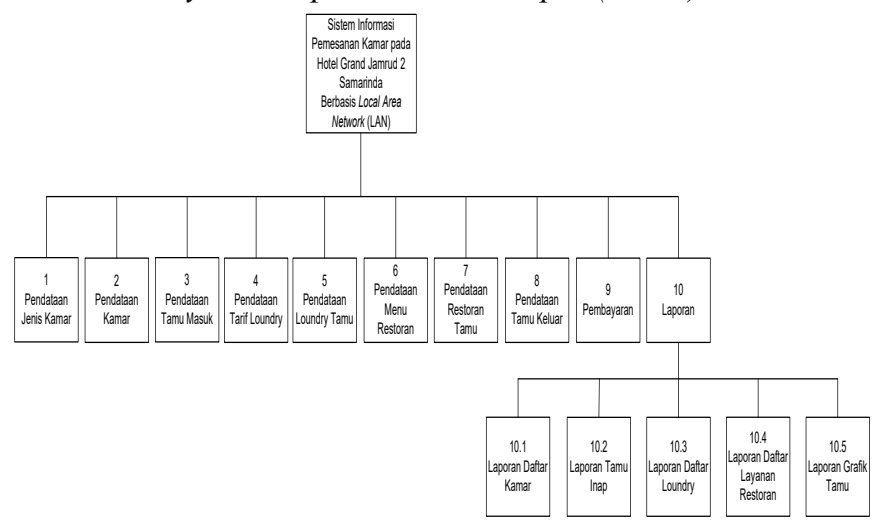

Fig. 10. HIPO of hotel reservation system

\section{G. Entity Relationship Diagram (ERD)}

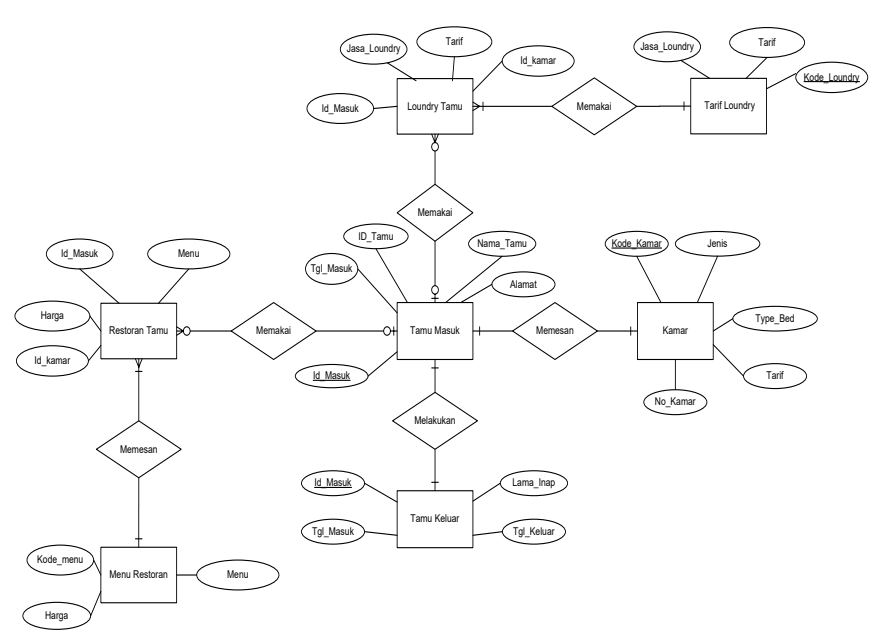

Fig. 11. ERD of hotel reservation system

\section{H. Prototyping of Network Design}

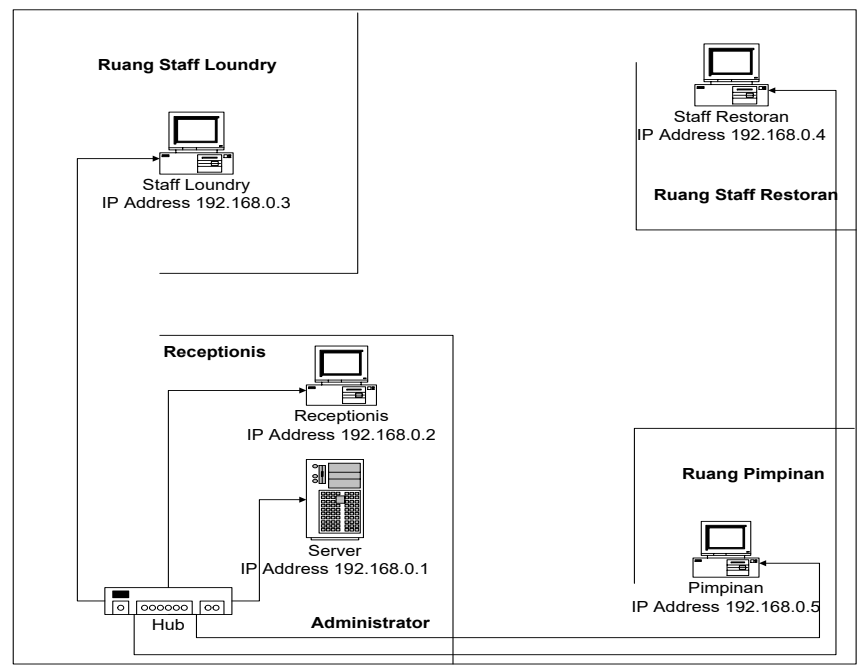

Fig. 12. Prototyping of network design
Prototyping of Hotel Network Design consists of five; computers, namely server, receptionist computer, laundry staff computer, restaurant computer staff, and leader's computer are placed in each room. All computers are connected each other in a network using a hub. Network topology to be used is star topology.

\section{Database Structure}

TABLE II. ROOM TABLE

\begin{tabular}{|l|l|c|c|}
\hline No & Field Name & Type & Size \\
\hline 1 & Code & Text & 3 \\
\hline 2 & Kind & Text & 10 \\
\hline 3 & Room_Number & Text & 3 \\
\hline 4 & Floor & Text & 1 \\
\hline 5 & Price & Currency & \\
\hline 6 & Type_bed & Text & 7 \\
\hline
\end{tabular}

TABLE III. GUEST_IN TABLE

\begin{tabular}{|l|l|c|c|}
\hline No & Field Name & Type & Size \\
\hline 1 & Code & Text & 7 \\
\hline 2 & Guest_Id & Text & 20 \\
\hline 3 & Guest_Name & Text & 35 \\
\hline 4 & Birth_Place & Text & 20 \\
\hline 5 & Birth_Date & Date/Time & \\
\hline 6 & Gender & Text & 9 \\
\hline 7 & Address & Text & 100 \\
\hline 8 & City & Text & 35 \\
\hline 9 & Room_Number & Text & 3 \\
\hline 10 & DateIn & Date/Time & \\
\hline 11 & DateOut & Text & 5 \\
\hline
\end{tabular}

TABLE IV. GUEST_OUT TABLE

\begin{tabular}{|l|l|c|c|}
\hline No & Field_Name & Type & Size \\
\hline 1 & Code_Out & Text & 7 \\
\hline 2 & Guest_Id & Text & 20 \\
\hline 3 & Guest_Name & Text & 35 \\
\hline 4 & Room_Code & Text & 3 \\
\hline 5 & DateIn & Date/Time & \\
\hline 6 & DateOut & Text & 5 \\
\hline 7 & TimeStay & Text & 2 \\
\hline
\end{tabular}

TABLE V. USER TABLE

\begin{tabular}{|c|l|c|c|}
\hline No & Field Name & Type & Size \\
\hline 1 & User & Text & 10 \\
\hline 2 & Password & Text & 10 \\
\hline
\end{tabular}

\section{J. System Application}

This hotel reservation system is a system that can be used by many people or many parts together so that the system is also a network-based system. This system will connect the server with users who act as a client. Both types of users will be interconnected in a single scope of the system. For hotel reservation system applied in the field used scheme Star network as follows. 


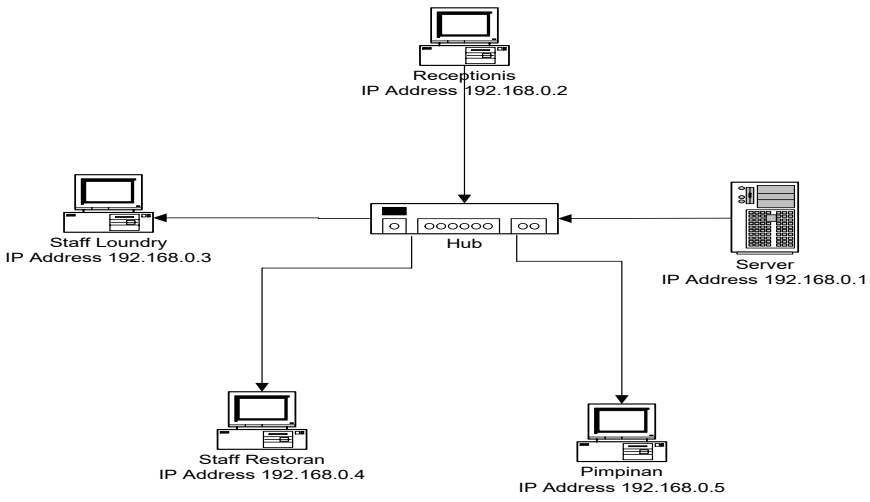

Fig. 13. Star Network of hotel reservation system

Because the system used server-based network server then required a connection between computers. There are several things that must be considered in the connection settings between computers are as follows.

- All drivers of computer hardware must be properly installed such as network card, network cable and connector.

- After all the drivers and installed, then next set the protocol TCP / IP (Transmission Control Protocol / Internet Protocol) each computer.

The steps of setting the TCP / IP protocol are as follows.

- Open windows explorer program and enter into control panel menu and select network connection.
- Select TCP / IP in the netwok dialog box then click properties.

- Next input IP Addres and subnet mask.

- Once the IP Address is stored, continue by filling the IP Address on another computer with the same NET ID and different Host ID.

- After all IP Address is stored, then all the computers have been connected in one network system.

\section{CONCLUSIONS}

This hotel reservation system is an application that can be used to facilitate the hotel room reservation including guest input data input, guest input data out, and payment transaction process, printing report of list room, and report guest list of stay. Due to this system has implemented a network-based system it will make it easier for each part in performing their tasks quickly and precisely.

\section{REFERENCES}

[1] R. Bemile, "Online Hotel Reservation System," International Journal of Innovative Science, Engineering \& Technology, vol. 1 Issue 9, November 2014, 2014.

[2] Hirschmann. (2001). Basics manual Local Area Network (LAN), Hirschmann Electronics GmbH \& Co.

[3] M. Ford. (1997). Internetworking Technologies Handbook.

[4] R. G. Sabale and A. R. Dani, "Comparative Study of Prototype Model For Software Engineering With System Development Life Cycle," IOSR Journal of Engineering (IOSRJEN), vol. 2, Issue 7(July 2012), pp. 21 $24,2012$.

[5] J. L. Whitten, L. D. Bentley, and K. C. Dittman. (2004). Systems Analysis and Design Methods.

[6] R. J. Robbins. (1995). Database Fundamentals. 